\title{
The Tubulin Superfamily in Archaea
}

\author{
Christopher H. S. Aylett ${ }^{1 \cdot}$ \& Iain G. Duggin²** \\ 1. Department of Biology, ETH Zurich, Zürich, ZH, Switzerland. \\ (christopher.aylett@mol.biol.ethz.ch)
}

2. The ithree institute, University of Technology Sydney, NSW, 2007, Australia. (Iain.Duggin@uts.edu.au)

*Address correspondence to: Iain.Duggin@uts.edu.au

\begin{abstract}
In comparison with bacteria and eukaryotes, the large and diverse group of microorganisms known as archaea possess a great diversity of cytoskeletal proteins, including members of the tubulin superfamily. Many species contain FtsZ, CetZ and even possible tubulins; however, some major taxonomic groups do not contain any member of the tubulin superfamily. Studies using the model archaeon, Halferax volcanii have recently been instrumental in defining the fundamental roles of FtsZ and CetZ in archaeal cell division and cell shape regulation. Structural studies of archaeal tubulin superfamily proteins provide a definitive contribution to the cytoskeletal field, showing which protein-types must have developed prior to the divergence of archaea and eukaryotes. Several regions of the globular core domain the "signature" motifs - combine in the 3D structure of the common molecular fold to form the GTP-binding site. They are the most conserved sequence elements and provide the primary basis for identification of new superfamily members through homology searches. The currently well-characterised proteins also all share a common mechanism of GTP-dependent polymerisation, in which GTP molecules are sandwiched between successive subunits that are arranged in a head-to-tail manner. However, some poorly-characterised archaeal protein families retain only some of the signature motifs and are unlikely to be capable of dynamic polymerisation, since the promotion of depolymerisation by hydrolysis to
\end{abstract}


GDP depends on contributions from both subunits that sandwich the nucleotide in the polymer. 
Archaea are a large and diverse group of microorganisms known for their remarkable metabolic diversity and capacity to thrive in harsh environments. They represent one of the three domains (or superkingdoms) of life, and have distinct relationships with eukaryotes and bacteria. They share a closer phylogenetic relationship to eukaryotes than bacteria, because the primary biosynthetic machineries needed in all cells - for DNA, RNA and protein synthesis-are relatively closely related in eukaryotes and archaea compared to the distantly related machineries found in bacteria. On the other hand, archaea and bacteria share a similar prokaryotic cellular organisation lacking the membrane-bound organelles and nucleus of the larger and more complex eukaryotic cells. Archaea also have unique and interesting characteristics, including alternative metabolic pathways, different membrane lipid and cell envelope structures, and archaea-specific families of cytoskeletal proteins. These organisms offer great potential to reveal new insights into the functions, evolution and diversity of cytoskeletal systems.

Whereas some proteins relating to basic cellular structure and function have been conserved in most prokaryotes since the early divergence of the archaeal and bacterial lineages (e.g. FtsZ), or in other cases acquired from one another later, recent studies have also identified archaeal homologs of proteins fundamental to eukaryotic cell structure and function. These include, among others, the primary filament-forming proteins of the cytoskeleton, actin (Chapter XX) and tubulin, and certain proteins of the Endosomal Sorting Complexes Required for Transport (ESCRT-III), which is a membrane remodelling system involved in cell division in Crenarchaeota (see Chapter YY). Some of these protein families must have developed prior to the divergence of archaea and eukaryotes, with some lineages of archaea retaining these where function has dictated.

Proteins from the tubulin superfamily share a common molecular fold related to GTP-binding. In all superfamily members, there are several regions within the globular core domain - the "signature" motifs - that combine in the $3 \mathrm{D}$ structure to form the GTP-binding site. These are the most conserved sequence elements and provide the primary basis for identification of new superfamily members through homology searches. Across diverse superfamily members 
characterized so far, these proteins also share a common mechanism of GTPdependent polymerization, in which GTP molecules are sandwiched between successive subunits that are arranged in a head-to-tail manner. However, some poorly characterised archaeal protein families described further below are unlikely to be capable of this mode of polymerisation, and retain only some of the signature motifs of the core domain. Depolymerisation is favoured upon hydrolysis to GDP, which can only occur through the contributions of both subunits that sandwich the nucleotide in the polymer. Regulated polymerisation and depolymerisation provide the basis for the assembly and movement of structural filaments that are the foundation of many cytoskeletal systems across the three domains of life.

The superfamily includes the numerous tubulin families that function to establish the microtubule-based cytoskeleton in eukaryotes, the FtsZs that provide the foundation for the bacterial, archaeal and some eukaryotic organelle division machineries, and several diverse relatives involved in microbial plasmid and viral segregation processes or with currently unknown functions.

\section{Distribution, diversity and functions of the tubulin superfamily in archaea}

Members of the tubulin superfamily are widespread in archaea. Fig. 1A shows a phylogenetic tree of the tubulin superfamily in the currently known major archaeal taxa, with representative groups of diverse FtsZ proteins (from bacteria and plants) and tubulins (eukaryotes) also included for comparison. A great diversity of archaeal homologs have now been identified. Despite this, archaeal tubulin superfamily proteins generally show a more limited species distribution amongst the sub-groups of Archaea, when compared to the near-ubiquity of FtsZ and tubulin within Bacteria and Eukaryota, respectively. Fig. 1B shows particular homologs of the named families that have been identified in the indicated archaeal taxa. Some archaea do not possess a clear tubulin superfamily homolog, including the major groupings of the Crenarchaeota, which use ESCRT-III (CdvB) based cell 
division machineries instead [27,54]. Many other archaea encode multiple tubulin superfamily homologs. Several major taxa of archaea contain members of a third family of cellular tubulin-superfamily proteins, called CetZ, whose members are clearly distinguishable from FtsZ and tubulin, and they have so far been found to control archaeal cell shape. Some archaea also possess the highly divergent FtsZlike (FtsZL1) proteins, and other non-canonical members, of currently unknown function (Fig. 1A, grey branches).

\subsection{Archaeal FtsZ}

FtsZ proteins are tubulin-superfamily proteins whose primary function is in forming the contractile ring for division of prokaryotic cells (bacterial and archaea), and organelles (specifically plastids, and mitochondria of some protists). They are the most wide-spread of the major groups of the tubulin superfamily, and generally show the greatest sequence similarity across diverse taxa (Fig. 1A). We refer to FtsZ-family proteins as those that sit within the branches indicated in Fig. 1A, for which there is evidence that they are involved in division, i.e. FtsZ (bacterial type, yellow), FtsZ1 (archaea, orange) or FtsZ2 (archaea, red).

\subsubsection{Identification of FtsZ in archaea}

The first tubulin superfamily proteins identified in archaea were from the FtsZ family. DNA hybridization approaches were used to clone ftsZ genes from two model species from the Halobacteria Class, Haloferax volcanii [64] and Halobacterium salinarum [34]. At the same time, isolation of GTP-binding proteins from cell lysates was used to identify an FtsZ from Pyrococcus woesei [9], a hyperthermophilic archaeon from the Class Thermococci. Not long after these discoveries, the first complete archaeal genome sequence, of Methanocaldococcus jannaschii, revealed that there were two ftsZ genes present in this organism [11], in contrast to most bacteria, which contain only one ftsZ. More recent genome se- 
quencing has confirmed that many archaea with FtsZ have two homologs from separate families called FtsZ1 and FtsZ2 [62] (Fig. 1A). The original clones of ftsZ from $H$. volcanii [64] and P. woesei [9] were FtsZ1 family members, whereas the gene originally isolated from $H$. salinarum [34] happened to be this organism's FtsZ2 family member.

Some archaea have only one FtsZ family member (Fig. 1A), including species of the Euryarchaeota Classes Methanobacteria and Methanopyri, as well as recently described candidate phyla Lokiarchaeota [58], Hadesarchaea [8], Thorarchaeota [57], and certain groups of the Thaumarchaeota (Candidatus Caldiarchaeum and Nitrososphaera)[44,68]. Furthermore, FtsZ has not been specifically identified in the Crenarchaeota, Bathyarchaeota (also known as the Miscellaneous Crenarchaeota Group (MCG) organisms [65]), and the majority of sequenced species of Thaumarchaeota. Some of these species encode divergent relatives with unknown functions (discussed in Section 1.2.2). In these organisms, the ESCRTIII-based system, first characterized in Crenarchaeota [27,55] is probably required for cell division $[40,48]$.

\subsubsection{Biochemical activities of archaeal FtsZ}

In the original study of $H$. volcanii FtsZ1, a recombinant tagged version of the protein was overproduced in E. coli and purified [64]. GTPase activity of this protein was demonstrated in buffers containing at least $2 \mathrm{M} \mathrm{KCl}(3 \mathrm{M} \mathrm{KCl}$ showed greater activity). The GTPase specific activity was dependent on FtsZ1 concentration ( $>200 \mu \mathrm{g} / \mathrm{mL}$ ), suggesting that self-association (polymerisation) is required for GTPase activity, as seen with bacterial FtsZ and eukaryotic tubulin. Work with Haloarcula japonica FtsZ1 revealed that polymerization in a sedimentation assay was dependent on GTP and at least $2.25 \mathrm{M} \mathrm{KCl} \mathrm{[47].} \mathrm{In} \mathrm{both} \mathrm{studies,}$ it was found that $\mathrm{NaCl}$ could not substitute for $\mathrm{KCl}$. Potassium is the predominant intracellular cation utilized at high concentration by haloarchaea for survival in hypersaline environments, so haloarchaeal proteins are well adapted to these conditions [51]. Potassium is also specifically required for efficient GTPase activity 
of other bacterial and archaeal FtsZ proteins, although generally at much lower concentrations in non-halophiles [35,37].

FtsZ homologs from thermophilic archaea have also been characterized. After the original identification of $P$. woesei FtsZ1, in which GTP binding was demonstrated [9], the M. jannaschii FtsZ1 protein became a focus for biochemical characterization after its crystal structure was solved [28]. This allowed some of the first high-resolution biochemical analysis of FtsZ proteins generally [29,30,17,5,21], outlined in part 2 of this chapter. Other thermophilic archaeal FtsZ proteins have also been investigated. One study isolated the ftsZ1 gene from the moderate thermophile Thermoplasma acidophilum [66]. Remarkably, expression of this gene in E. coli resulted in strong inhibition of cell division, which was attributed to interaction of the T. acidophilum FtsZ with the native FtsZ in E. coli and/or interference with its function. Another FtsZ-related protein was isolated from the hyperthermophile Thermococcus kodakaraensis [39], however, this protein is now recognised as a CetZ-family protein (Section 1.3).

\subsubsection{The role of archaeal FtsZ in cell division}

The first cytological evidence to suggest that archaeal FtsZ functions in cell division utilized immunofluoresence microscopy with $H$. volcanii. FtsZ1 was seen to localize at the mid-cell region, appearing as a ring around the middle of the cell that corresponded with mid-cell constrictions in cells that appeared to be dividing [64]. Similar results were obtained with the closely related Haloferax mediterranei, where it was also seen that constricted FtsZ1-rings were associated with cells of a variety of different shapes, including rods and the irregular plate-shaped cells that are common in these pleomorphic haloarchaea [49]. Recently, H. volcanii FtsZ1 tagged with GFP was observed in live cells [18], and the localization pattern was consistent with the earlier immunofluorescence results. FtsZ1-GFP was produced at a low level from a regulated gene promoter in a wild-type genetic background, which resulted in cells of normal size, indicating minimal disturbance to the normal cell division process by the presence of FtsZ1-GFP. Interestingly, 
almost all cells in a growing culture were found to have a mid-cell ring, indicating that this structure is maintained throughout the cell cycle. As most cells in these cultures do not contain noticeable division constrictions, FtsZ ring assembly occurs well in advance of constriction, similar to bacteria [60].

The fluorescence microscopy approaches revealed obvious similarities between the basic subcellular localization behaviour of archaeal and bacterial FtsZ, strongly implicating FtsZ in archaeal cell division. However, the original study of FtsZ in H. salinarum found that supplementary expression of ftsZ2 did not greatly affect cell division, since cell size was similar to the wild-type [34]. In contrast, overexpression of ftsZ in E. coli causes division defects that affect cell size [16]. The H. salinarum cells instead appeared pleomorphic, quite similar to the wild-type strains of some other haloarchaea, whereas wild-type H. salinarum is typically rod-shaped in laboratory culture. The basis for this morphological effect is yet to be determined, however, haloarchaeal cell shapes are generally sensitive to experimental conditions and it is possible that FtsZ overexpression does not have an inhibitory effect on division in all species, as it does in E. coli.

Direct evidence for the importance of FtsZ in archaeal cell division came only recently [18]. Expression of a point mutant of $H$. volcanii ftsZ1 (D250A), which targets the GTPase activation region (T7 loop) essential for the normal polymerization-depolymerisation cycle in tubulin superfamily proteins $[53,56]$, caused the hallmark cell division defect, in which cells cease division but continue to grow, resulting in the generation of extremely large cells (e.g. Fig. 2B). Thus, a functional GTPase activation domain in FtsZ1 is essential for normal cell division.

The genetic amenability of haloarchaea, particularly $H$. volcanii [2], and the availability of functional fluorescent fusion proteins $[18,52]$ combined with their relatively large, flattened cell morphologies, show that these organisms are excellent models for understanding archaeal cell division, morphology, and cell biology generally [18]. Future studies with this system are anticipated to provide significant insights into functional diversity of cell division and structure, providing a much needed contrast with the well-studied bacterial systems. This is ex- 
pected to highlight the most fundamental features critical to both systems, and reveal a very different FtsZ-based system for prokaryotic cell division.

\subsection{Archaeal tubulin and FtsZ-like homologs}

As may be seen in Fig. 1A, archaea house a variety of deeply-branching tubulin superfamily members, many of which are not classifiable into the FtsZ, tubulin or CetZ families, and their functions are essentially unknown. Many of these proteins have only recently been identified owing to the recent vast increase in genome sequence availability, brought about by major advances in high-throughput environmental DNA sequencing.

\subsubsection{The FtsZ-like group 1 homologs (FtsZL1)}

Members of the unusual FtsZ-like group 1 (FtsZL1) were identified in certain bacteria and archaea on the basis of homology to the GTP-binding core (N-terminal) domain, which includes the signature motifs of the tubulin superfamily [33]. There are only a small number of known FtsZL1 sequences in archaea, although they may be found in diverse species including the Methanomicrobia, Halobacteria and Thermococci Classes. These proteins form a phylogenetic subgroup distinct from the related bacterial FtsZL1 proteins, suggesting that recent horizontal transfer between domains is an unlikely mechanism to explain their patchy distribution.

Interestingly, a transposon-interrupted FtsZL1 gene was identified in Sulfolobus solfataricus (SSO1376), representing the first tubulin-superfamily relative found in the Crenarchaeota [33]. Another FtsZL1 member (undisrupted) in the Crenarchaeote Acidilobus saccharovorans was recently sequenced (Uniprot D9PZB8). Both Crenarchaeote FtsZL1 proteins have unusually-long homologous $\mathrm{N}$-terminal extensions to the core GTP-binding domain, suggesting that these have a particular (unknown) function in Crenarchaeota FtsZL1. 
The domain arrangement of FtsZL1 proteins more generally is highly unusual amongst the tubulin superfamily, as the central GTPase activation region and C-terminal domain, normally involved in polymerization (Fig. 4), are replaced with a much larger and unrelated domain of unknown structure and function. Thus, the capacity of these proteins for polymerization and GTP hydrolysis is currently unknown. A noticeable conservation of the FtsZL1 genomic neighbourhood across diverse species led to the hypothesis that these proteins are involved in membrane remodelling processes in conjunction with the products of the linked genes [33].

\subsubsection{Thaumarchaeota tubulin-superfamily members and other deeply-branching archaeal homologs}

The Thaumarchaeota show quite a complex distribution of tubulin-superfamily proteins, with specific homologs of FtsZ and tubulin seen in some species, and another family called "Thaumarchaeota FtsZ" [67] present in most of the major taxa of the Thaumarchaeota (Fig. 1). Despite the name currently used for this family, the Thaumarchaeota-FtsZ proteins are distinct from the major FtsZ, CetZ and tubulin branches, and they are at least as far removed from the FtsZ family as another small group of proteins from the Korarchaeota (Fig. 1A). In addition to these, Korarchaeota possess specific FtsZ family sequences (but not ESCRT-III), suggesting that Korarchaeota division is based on FtsZ, and that the other deeplybranching homologs in these organisms may have other non-cell-division roles. We would suggest that newly identified tubulin-superfamily groups, such as Thaumarchaeota-FtsZ, be renamed where appropriate once a prototypical member has a clear biological role determined, so that the term "FtsZ" is limited to proteins that primarily function in division.

The Thaumarchaeota-FtsZ group shows significant sequence divergence in the GTP-binding T4-loop, with a consensus of AGKAG in this region instead of the superfamily consensus GGGTG. Most Thaumarchaeota-FtsZ proteins also appear to completely lack the catalytic residues of the T7 loop required for GTP hy- 
drolysis. Thus, the normal mode of polymerization and depolymerisation is very unlikely to occur in these proteins. Consistent with this, the Thaumarchaeota-FtsZ protein in Nitrosopumilus maritimus does not appear to be involved in cell division, as it was localized diffusely in these cells, whereas the CdvB (ESCRT-III) homolog was located at mid-cell (division) sites [48]. Furthermore, an N. maritimus FtsZ-GFP fusion showed diffuse localization when expressed in yeast, whereas CdvB-GFP formed obvious filaments [40]. Interestingly, when the unusual T4 loop sequence of $N$. maritimus FtsZ was replaced by the E. coli FtsZ T4 loop, this resulted in localization as foci and short filaments in yeast, much like the localization pattern of the E. coli FtsZ (GTPase-deficient mutant) in this system [40]. The substituted N. maritimus FtsZ might therefore have gained nucleotide binding but not hydrolysis capacity, promoting polymerization and assembly into localized structures. The wild-type Thaumarchaeota-FtsZ proteins therefore might not bind/hydrolyse GTP or polymerise, so they remain one of the more mysterious groups in the tubulin superfamily.

Another group of tubulin-superfamily proteins in Thaumarchaeota, called artubulin, was identified in the genomes of two members of the genus Nitrosoarchaeum, in the order Nitrosopumiales (Fig. 1B)[67]. Thaumarchaeota-FtsZ genes are also present in these organisms. The artubulins form a sister group to the eukaryotic tubulin family (Fig. 1A), raising questions surrounding the origin of this family within the eukaryotic lineage. The artubulin genes are located adjacent to a component of the ESCRT-III machinery, hinting at a possible role of artubulin in ESCRT-III mediated division in these organisms [67]. Future functional studies of artubulins may provide insights into the early evolutionary events that led to the expansion of tubulin-superfamily roles in the Eukaryota/Archaea lineage.

There are a number of other tubulin-superfamily proteins in archaea that are currently uncharacterized (grey branches, Fig. 1A). These could be expected to have specialized roles, and of particular note are the numerous highly-divergent tubulin-superfamily members present in Haloquadratum walsbyi [10,12]. This organism forms remarkably thin, square cell shapes that are maintained in this shape during cell division $[63,59]$. The shapes would appear to provide a substantial se- 
lective advantage in their hypersaline lake environments [12]. One might predict that the divergent tubulin-superfamily proteins form cytoskeletal structures required for generating and maintaining the square shapes, while the FtsZ and CetZ proteins that also exist in this organism may have roles in cell division and morphological changes, respectively. The future study of the cytoskeletal systems of this and related organisms is a fascinating prospect.

\subsection{The Euryarchaeota CetZ family of proteins}

The CetZ family were first recognised as a phylogenetically distinct group, originally called FtsZ paralog 3 (FtsZ3), after the cloning and sequencing of an ftsZ homolog from Thermococcus kodakaraensis [39], a hyperthermophilic species of the Class Thermococci. The purified protein showed GTPase activity and polymerisation that increased continuously with temperature, up to $90^{\circ} \mathrm{C}$, which is near the growth optimum of this organism. The protein sequence showed high similarity to one of three homologs in the available genome sequence of $P y$ rococcus horikoshii (Class Thermococci) [22], and was only distantly related to the two other homologs, FtsZ1 and FtsZ2. Other members of the CetZ family were then noticed in the genomes of Pyrococcus abyssi [15] and Archaeoglobus fulgidus (Class Archaeoglobi) [23], raising the possibility that the multiple conserved paralogs in these archaea could have differing cytoskeletal roles [20]. A thorough survey of tubulin-superfamily sequences [62] further identified two cet $Z$ genes in the genome of Halobacterium NRC-1 (Class Halobacteria) [41]. It was also observed that the Thermococci CetZ (FtsZ3) proteins branch from near the base of the group [62]. The large quantity of sequence data now available has shown that CetZ proteins are present in many other diverse archaea from the phylum Euryarchaeota, including the Class Methanomicrobia [18]. CetZ proteins form a distinct branch (family) within the tubulin-superfamily (Fig. 1A).

The first in vivo functional study of members of the CetZ family found that they control cell morphology in H. volcanii [18]. The family was named cell- 
structure-related Euryarchaeota tubulin/Fts $\underline{Z}$ homologs (CetZ) to reflect the noncell-division role seen for $H$. volcanii CetZ1. cetZ genes are especially abundant in genomes of Halobacteria (e.g. six paralogs in $H$. volcanii), although the number of paralogs varies amongst related species. Some of the Haloarchaeal CetZ proteins cluster phylogenetically into several distinct orthologous groups, suggesting that proteins in each group may have a common function in these species (Fig. 1A). The most conserved of these is the CetZ1 group, which was present in all species of Halobacteria analysed [18], and members typically shared $\sim 80 \%$ sequence identity in this Class. CetZ2 orthologues were identified in many of these. The $H$. volcanii CetZ3-6 proteins were located in weakly-clustered groups and might be expected to have more specific or redundant roles in this species. Generally, the Methanomicrobia and Halobacteria Classes processed multiple CetZ homologs, whereas the Archaeoglobi and Thermococci carry one CetZ each. CetZ has so far only been identified in species that also contain FtsZ (Fig. 1B).

The function of CetZ proteins was studied in the model organism $\mathrm{H}$. volcanii [18]. This species is described as pleomorphic [38], displaying a variety of cell sizes and shapes in complex growth medium (e.g. Hv-YPC [1]). However, as in many haloarchaea, the cells remain uniformly thin $(\sim 0.4 \mu \mathrm{m})$ during growth and division, growing either two-dimensionally (the plate morphotype) or onedimensionally (the rod morphotype). H. volcanii cells lye on their flat, larger surfaces when placed on a gel-pad, and they can be grown for many generations on a Hv-Cab gel-pad; the relatively large, flat cells are well-suited for visualization by fluorescence microscopy (e.g. by using GFP or mCherry) and other approaches including 3D-SIM and electron cryotomography [18]. Time-lapse imaging of live cells showed that they can transition between the two basic modes of cell growth, and some cells appear to actively narrow during the plate-to-rod transition [18]. The CetZ1 protein was found to be essential for the development of the rod morphotype, which was needed for efficient swimming motility [18].

When $H$. volcanii is in steady-state growth (mid-log growth for $>10$ generations) in a defined medium, "Hv-Cab" (i.e. Hv-Ca [1] with additional traceelements [18]), it grows consistently as the plate morphotype [18]. However, 
moderate overexpression of CetZ1, via the use of the finely-regulated tnaA gene promoter [3,25], caused many of the cells to adopt an obvious rod morphology. Interestingly, overexpression of the CetZ1.E218A mutant (to prevent GTPase activity and filament depolymerisation) caused the cells to adopt a very blocky, jagged morphology instead (a similar phenotype was seen with CetZ2.E212A [18]). This appears to be caused by hyperstable filaments of CetZ1.E218A, since a GFP fusion protein to this mutant localized very stably to the cell envelope at the stalklike projections and inward puckers seen in these cells. In contrast, CetZ1-GFP showed very dynamic/patchy or diffuse localization in the log-phase plate cells. During rod development, however, CetZ1-GFP sometimes appeared as faint, dynamic filaments aligning with the direction of rod cell development, suggesting that such cytoskeletal structures are specifically related to rod development. It was concluded that CetZ1 forms a key part of an archaeal cytoskeletal system involved in regulation of cell morphology, and represents a prototypical member of this family of cytoskeletal proteins.

The great amenability of $H$. volcanii to genetic manipulation [2], combined with its ease of growth, controllable cellular differentiation processes, and excellent properties for many live-cell microscopy approaches [18] show that it is an excellent model for further studies into the archaeal cytoskeleton and cell biology.

\section{Structures of tubulin superfamily proteins from archaea}

In this section, we describe the original role structural studies of archaeal tubulinsuperfamily proteins have played in helping identify and characterize cytoskeletal proteins in archaea and other organisms, and we describe the known archaeal protein structures and aspects of the structure-function relationship that have been obtained from these studies. Finally, we compare structures of the recently-described CetZ family to the other major groups of the tubulin superfamily. 
Archaeal proteins can provide technical advantages in structural studies, such as high thermostability, which in many cases have enabled new insights and clues into the structure and function of less amenable homologs in the intensively studied eukaryotic and bacterial species. An archaeal FtsZ featured prominently amongst the first high-resolution structural studies of the tubulin superfamily [28]. In this section, we will describe the general features of the protein structures that have been obtained from studies of archaeal tubulin superfamily proteins, and compare them with other members of the superfamily. Atomic-resolution structures that are currently available for archaeal tubulin superfamily proteins are listed in Table 1.

Table 1. Available crystal structures of archaeal tubulin superfamily proteins.

\begin{tabular}{|c|c|c|c|c|}
\hline Protein & Organism $^{a}$ & $P D B \operatorname{code}^{b}$ & Resolution & Notes \\
\hline FtsZ1 & M. jannaschii & $1 \mathrm{FSZ}[28]$ & $2.8 \AA$ & GDP-bound monomer \\
\hline FtsZ1 & M. jannaschii & 2VAP $[46]$ & $1.7 \AA$ & GDP-bound monomer (different crystal form) \\
\hline FtsZ1 & M. jannaschii & $1 \mathrm{~W} 58[45]$ & $2.5 \AA$ & $\mathrm{Mg}^{2+}$ and GMPCPP-bound ${ }^{c}$ monomer \\
\hline FtsZ1 & M. jannaschii & $1 \mathrm{~W} 59[45]$ & $2.7 \AA$ & Nucleotide-free dimer \\
\hline FtsZ1 & M. jannaschii & $1 \mathrm{~W} 5 \mathrm{~A}[45]$ & $2.4 \AA$ & $\mathrm{Mg}^{2+}$ and GTP-bound dimer \\
\hline FtsZ1 & M. jannaschii & $1 \mathrm{~W} 5 \mathrm{~B}[45]$ & $2.2 \AA$ & GTP-bound dimer \\
\hline FtsZ1 & M. jannaschii & $1 \mathrm{~W} 5 \mathrm{E}[45]$ & $3.0 \AA$ & W319Y mutant (GTPase defect), GTP-bound \\
\hline CetZ1 & H. volcanii & $4 \mathrm{~B} 46[18]$ & $1.9 \AA$ & GDP-bound monomer \\
\hline CetZ2 & H. volcanii & $4 \mathrm{~B} 45[18]$ & $2.1 \AA$ & GTP $\gamma \mathrm{S}$-bound protofilament (trimer) \\
\hline CetZ & M. thermophila & 3ZID [18] & $2.0 \AA$ & GDP-bound \\
\hline
\end{tabular}

${ }^{a}$ Full organism names: Methanocaldococcus jannaschii; Haloferax volcanii; Methanosaeta thermophila.

${ }^{b}$ Protein Data Bank identification code (www.rcsb.org). The associated reference is given in parentheses.

${ }^{c}$ GMPCPP is a GTP analog: Phosphomethylphosphonic Acid Guanylate Ester.

\subsection{FtsZ structures}

In 1998, an FtsZ protein from the archaeon Methanocaldococcus jannaschii became the first member of the tubulin superfamily to have its structure solved at high resolution by X-ray crystallography [28]. When compared to the electron crystallographic structure of polymerized tubulin reported in the same issue of $\mathrm{Na}$ - 
ture [43], debate as to the homology between FtsZ and tubulin ended-their structural folds were remarkably similar [42]. Thus, structural comparisons of these proteins from highly diverse organisms highlighted the usefulness of protein 3D structure in verifying weak homology identified through alignment of a limited number of sequences. Additional structures of the M. jannaschii FtsZ1 protein, including different crystal forms and ligand-bound states have helped provide further insights into protofilament formation and structural variability that underpin the basic functions of these proteins $[29,30,45,46]$.

The original structure of $M$. jannaschii FtsZ1 bound to GDP showed that it is a globular protein (39 $\mathrm{kDa})$ containing two closely associated but independently-folded domains [28,45], essentially the same as tubulin [43]. These proteins form a distinct superfamily of GTPases [42], which nevertheless retain some structural similarities to the Rossmann-fold di-nucleotide binding proteins and small GTPases of the Ras superfamily [28]. This similarity is seen in the Nterminal GTP-binding domain, which contains most of the secondary structural elements that establish the nucleotide-binding site (shown in green; Fig. 3). However, within this domain the tubulin superfamily is distinguished by the presence of four highly conserved loops (T1, T2, T3 and T4, on the upper surface as depicted in Fig. 3) that primarily engage the phosphates of the nucleotide, whist allowing another FtsZ subunit to bind to this surface in a "head-to-tail" orientation (i.e. via the lower surface of the additional subunit), sandwiching the GTP molecule [45]. Thus, both subunits supply residues required for GTP hydrolysis, explaining why GTP hydrolysis is polymerization-dependent [37].

The C-terminal domain (shown in purple; Fig. 3) is separated from the Nterminal GTP-binding domain by an important central region, consisting of the long H7 helix (yellow; Fig 2) followed by the T7/H8 region. Although not usually considered a distinct domain, this region nonetheless has the specific function of delivering key acidic residues to the GTP-binding region of the adjacent subunit, and instigates a nucleophilic attack at the gamma-phosphate of GTP, resulting in hydrolysis to GDP. The GTPase activation function of the H7/T7/H8 region has the important role of promoting filament disassembly and turnover [42]. 
Hydrolysis and filament disassembly are very important processes in the spatial regulation and cytomotive activity of cytoskeletal structures [32]. The early studies of M. jannaschii FtsZ1 raised the question of how hydrolysis leads to filament disassembly; a comparison of numerous crystal structures of FtsZ proteins, including several guanosine-nucleotide bound states of $M$. jannaschii FtsZ1, revealed no large conformational changes associated with hydrolysis [46]. Recent high-resolution electron cryo-microscopy (cryoEM) of tubulin in various states would suggest that hydrolysis causes a more subtle compaction around the region of the GTP-binding domain that surrounds the nucleotide's phosphate groups (i.e. the T1-T4 loops). This creates strain within the subunit and moderate conformational shifts that destabilize the longitudinal interaction between subunits [4]. It therefore appears that the flexible glycine-rich T1-T4 loops, which include the tubulin superfamily "signature" sequence (T4), sense the number of phosphates of the nucleotide and transduce this information to the whole molecule in order to promote disassembly. It remains to be determined whether this mechanism occurs in the same manner in FtsZ filaments, but the strong conservation of these loops would suggest it is very likely.

The presence and structure of the C-terminal domain (shown in purple; Fig. 3) is another defining characteristic of the tubulin superfamily amongst GTPases, and shows a conserved fold despite the very sparse sequence conservation amongst the superfamily's most diverse members. Crystal structures of $M$. jannaschii FtsZ1 dimers that sandwich GTP in the expected manner [45] showed that the C-terminal domain provides the majority of the contact surface area between subunits (Fig. 4C). This is also observed in the other families (Fig. 4). The C-terminal domain can also mediate lateral association of protofilaments $[4,18,36]$, fulfilling another essential characteristic of the superfamily - the ability to form bundles, sheets and tubes that build the cytoskeleton in its myriad of forms across the domains of life. One of the C-terminal domain's main purposes therefore appears to be to mediate self-association.

In addition to the two main domains of the globular fold of FtsZ, these proteins frequently have $\mathrm{N}$ - and $\mathrm{C}$-terminal extensions that have specific func- 
tions, generally in mediating interactions with other molecules. They protrude from the "front and "rear" surfaces of the molecule, respectively (as shown in Figs. 2 and 3), and are thus located on the two exposed surfaces of protofilament sheets. These regions are rarely fully resolved in crystal structures owing to their flexibility and lack of secondary structure in isolation. M. jannaschii FtsZ1 showed an N-terminal extension (which is generally absent in the tubulin or CetZ families) that has limited secondary structure apart from one helix at the very $\mathrm{N}$ terminus [28]. The function of this helix and remaining N-terminal extension is still unknown. The C-terminal extension in $M$. jannaschii FtsZ1 is a $\beta$-hairpin, comprising strands S11 and S12, beyond which the protein is typically extended without significant structural elements, and differs from the primarily helical Cterminal extensions seen in tubulin and CetZ proteins (discussed below). The length of the C-terminal extension is highly variable amongst different species and in bacteria this is thought to play an important role as a flexible spacer [14,19], while the more sequence-conserved few residues immediately preceding the $\mathrm{C}$ terminus bind other molecules with important roles in cell division [13]. In archaea, molecules that interact with FtsZ are unknown, however the conservation of the C-terminal few residues amongst other archaea would suggest that these residues have a common function in archaea too.

\subsection{CetZ structures}

Three crystal structures of CetZ family proteins recently solved [18] show some interesting similarities and differences to the FtsZ and tubulin family proteins that may eventually help to reconcile structure-function relationships in the superfamily.

\subsubsection{H. volcanii CetZ1}


The structure of CetZ1 from H. volcanii represents the prototypical CetZ, having been the first structure solved, and to date it is the only CetZ protein to which a definitive function in the regulation of cellular morphology has been ascribed [18]. The structure revealed CetZ1 in a monomeric state, with a GDP molecule that had co-purified retained in the active site. CetZ exhibits the two principle domains present in other tubulin superfamily proteins, however several notable modifications to the typical structural features are apparent. CetZ1, and indeed most CetZs, lack a N-terminal extension to the core GTP-binding domain. By comparison, an $\mathrm{N}$-terminal extension is generally present in FtsZ, but absent in tubulins. Within the GTP-binding domain, helix H6 is substantially shorter than usual, leaving only a single helical turn to form this section of the subunit-subunit interface (Fig. 4A). Helix H9, on the other hand, is considerably extended, increasing the size and prominence of the C-terminal domain. Furthermore, the nearby M-loop, which links S7 and H9 (Fig. 3A), is unresolved in the crystal structure but is unusually long in CetZ proteins compared to FtsZ and tubulins. The M-loop mediates lateral association of tubulin protofilaments within microtubules $[4,42,43]$, but is of unknown function in CetZ proteins. Finally, the C-terminal extension of CetZ1 is a long helix (H11), which aligns with the expected axis of polymerisation of protofilaments. These three regions of notable difference (i.e. H6, H9 and H11) compared to FtsZ and tubulin are also the only regions showing notable variations in structure that were observed between $H$. volcanii CetZ1 and CetZ2.

\subsubsection{H. volcanii CetZ2}

CetZ2 was crystallized and its structure solved bound to GTP $\gamma$ S [18], which resulted in the generation of a lattice incorporating crystallographic protofilaments. The subunit structure of $H$. volcanii CetZ2 is remarkably similar to CetZ1; the $\mathrm{C} \alpha$ r.m.s.d between the two structures is only $0.85 \AA$, as might be expected given their relatively high sequence similarity (51\% identical). CetZ2 protofilaments in the crystal lattice had a repeat unit of $43.2 \AA$, very similar to tubulin $(41.8 \AA$ ), and even closer to FtsZ and TubZ proteins (43.5 $\AA$ ). Furthermore, the subunit-subunit 
interface formed within CetZ2 protofilaments is very similar to that conserved within the tubulin and FtsZ groups, although it differs more from that in the TubZ proteins, which are tilted differently across the active site. This directly affects the conformation of protofilaments, and CetZ2 recapitulated the straight protofilaments typically seen in crystal structures of FtsZ and tubulin protofilaments. This contrasts some of the known bacterial TubZ protofilaments, which show twisted, helical arrangements [6]. The presence and degree of helical structure directly affects the higher-order filament or sheet/tube-like structures that are critical to the function of these proteins, and it will be informative to see the conformation of further CetZ filaments, ideally with methods that have minimal influence on the subtle quaternary arrangements important to tubulin superfamily proteins.

The C-terminal helix (H11) in CetZ2 occupies a similar space to that of the tubulins and TubZ proteins, and aligns almost perfectly with H11 from tubulin protofilaments (1.0 $\AA \mathrm{C} \alpha$ r.m.s.d, CetZ2 C-terminus versus tubulin C-terminus). H11 also extends along the surface, generally in the longitudinal direction of polymerization, and contacts the base of the adjacent subunit, similar to tubulins and TubZ proteins. Although regions of the C-terminal tail from H11 onwards are not fully resolved in the structure, it would appear possible that the H11 helix of CetZ proteins may continue along the surface of the adjacent subunit in a manner similar to the TubZ proteins, with H11 filling the space that would otherwise be occupied by $\mathrm{H} 12$ in the multi-helix folded C-terminal tails seen in eukaryotic tubulin protofilaments.

Interestingly, the CetZ2 protofilaments were arranged as crystallographic sheets, with lateral contacts between protofilaments generally comparable to the lateral interactions of tubulin in the microtubule wall and FtsZ lateral associations, suggesting that such interactions might be biologically relevant in CetZ proteins too. The loops of C-terminal domain, including an extended S7-H9, and those on the same face of the GTPase domain, including the altered loop H1-S2 as well H3. Further support for the significance of these contacts comes from the observation that the lateral crystal contacts within the more distantly related Methanosaeta thermophila CetZ crystal structure recapitulate this interaction almost perfectly 
(2.6 $\mathrm{A} \mathrm{C} \alpha$ r.m.s.d over adjacent chains; H. volcanii CetZ2 versus M. thermophila CetZ).

The aforementioned sheets within CetZ2 crystals are bridged by the Cterminal tail of the protein. An interesting observation, however, was the association of the C-terminal amino acids of one subunit within a pocket abutting helix $\mathrm{H} 7$ of an adjacent subunit. This pocket in tubulin is where the anticancer drug, taxol, binds to $\beta$-tubulin in protofilaments. The concomitant slight gap in the closely related CetZ1 crystal structure, in an unpolymerised state, is closed somewhat by a small rotation of the activation domain and remains unoccupied; it is not possible to say from our results whether protofilament formation is responsible for this rotation and intrusion of the C-terminal peptide, or whether it might be a constitutive difference between the folds of the two CetZ proteins, however we note that a similar rotation of the activation domain was observed in microtubules stabilised in the presence of taxol or GTP analogues. It is yet to be determined whether such docking of the C-terminal residues on the adjacent protofilament sheet of CetZ2 has any relevance to the physiological form and function of CetZ proteins.

\subsubsection{Methanosaeta thermophila CetZ}

The structure of a divergent CetZ homolog, which branches from near the root of the tubulin superfamily phylogenetic tree (Fig. 1), was also determined [18]. Superimposition of this $M$. thermophila CetZ on $H$. volcanii CetZ2 leads to an overall $\mathrm{C} \alpha$ r.m.s.d of only $1.8 \AA$, and the structural features observed within both chains are also very well conserved from those in the two $H$. volcanii CetZ structures. The only clear difference between the $M$. thermophila and $H$. volcanii proteins is the substantial extension of the carboxy-terminal helix H11 resolved in $M$. thermophila CetZ and a slight shortening of helix H9, the latter being more like the $\mathrm{H} 9$ of other tubulin superfamily proteins. Although there are many small surface differences between the proteins from the two organisms, the deviation between surface loops is extremely low, supporting the conclusion that the proteins 
should be grouped cleanly into the single (CetZ) family. Since these proteins branch from near the base of the superfamily tree, it will be useful to determine the function of M. thermophila CetZ, or similar "non-canonical" CetZ proteins, to determine whether these proteins have functions in cell shape regulation like the H. volcanii CetZ1 and CetZ2 proteins [18] or as yet unknown functions.

\subsection{Structural comparisons of CetZs with other tubulin superfamily proteins}

As noted above, the archaeal FtsZ and CetZ families exhibit many features in common with eukaryotic tubulins as well as bacterial FtsZ and TubZ proteins. Here, we will compare structures of CetZ proteins to those of the FtsZ protofilament structure from Staphylococcus aureus [61] (PDB code 4DXD), the taxol/Zinc-stabilised sheets of Bos taurus tubulin protofilaments [31] (PDB code 1JFF), and Bacillus thuringiensis [6] and Pseudomonas aeruginosa bacteriophage ФKZ [7] TubZ protofilament structures (PDB codes 2XKB and 3ZBQ respectively) as prototypes.

\subsubsection{Comparison of CetZ and FtsZ}

The CetZ protein structures show several notable structural features specifically in common with FtsZ. In particular, the conformation of the key $\gamma$-phosphate contact loop T3 and proximal regions (S3, H3) in both H. volcanii CetZ1 and CetZ2 is almost identical to that in the majority of FtsZ structures resolved (C $\alpha$ r.m.s.d 1.1 $\AA$ FtsZ against CetZ1). The conformation of loop T5 is also strongly conserved between these two groups, although it typically exhibits lower overall deviation in many cases, and the numerous surface loops of the CetZ proteins are closer in conformation to those in FtsZ than tubulins. The overall backbone difference between CetZs and FtsZs (overall C $\alpha$ r.m.s.d $2.9 \AA$ for FtsZ and CetZ1) is also significantly lower than that observed on comparison with tubulins (overall $\mathrm{C} \alpha$ 
r.m.s.d 3.6 $\AA$ for $\alpha$-tubulin and CetZ1). However, as we note below, a series of conserved catalytic residues shared between the tubulins and CetZ proteins provide a counterargument to this narrative.

\subsubsection{Comparison of CetZ and tubulin}

The tubulins are the most well understood proteins in the superfamily. They build the microtubule network present in all known eukaryotes, which is a major component of the cytoskeleton. Microtubules control cellular organisation and have well-known role in providing the spindle for chromosome segregation. The polymerising unit for tubulin includes two different monomers, $\alpha$ - and $\beta$ tubulin, which are pre-assembled around a molecule of GTP which is bound essentially irreversibly within a catalytically inactive subunit-subunit interface. In addition to the canonical N-terminal GTP-binding and C-terminal domain structure, tubulins incorporate a distinct $\mathrm{C}$-terminal extension comprising a helical hairpin ( $\mathrm{H} 11$ and $\mathrm{H} 12)$ that provides the binding platform for processive motor proteins, and several notable surface loops (H1-S2, H2-S3, M- and N-loops) that form the lateral interactions which stabilise the contacts between the thirteen protofilaments incorporated into each cylindrical microtubule [31].

In the CetZ proteins, the active site shows great similarity to that found in $\alpha$-tubulin. Guanine base recognition is achieved through an asparagine residue protruding from $\mathrm{H} 7$ in all three CetZ structures, a similar situation to that in tubulins and bacterial TubZ proteins (described below), whereas the equivalent residue is an aspartate in the FtsZs. Furthermore, the essential catalytic carboxylate within T7/H8, which activates water for $\gamma$-phosphate hydrolysis, is also provided by a glutamate residue in CetZ and tubulin, but is an aspartate in FtsZ. All three CetZ structures share a glutamine residue at the apex of loop T1 which forms an important protofilament contact with loop T7 in tubulin protofilaments, but which is absent in the known FtsZ structures and replaced in many TubZs. Finally, several regions of the CetZs form loops extending from the core fold, the location of which are identical to those responsible for lateral interactions between tubulin 
protofilaments (S7-H9 in CetZ2, and H1-S2 in M. thermophila CetZ). However, the loop structures are not homologous between CetZs and tubulins, suggesting that they may differ substantially in function.

\subsubsection{Comparison of CetZ and TubZ}

The TubZ group of proteins represent some of the most diverse members of the established tubulin superfamily, and they cannot clearly be classified into their own distinct subgroup. Two functional sub-classes exist, one responsible for partitioning plasmids and prophages within species of Gram-positive Bacilli, and the other associated with bacteriophage of the genus Pseudomonas and closely-related bacteria $[24,26]$. TubZ proteins typically have a long $\alpha$-helical C-terminal extension that makes extensive contacts with the adjacent subunit and is also believed to be responsible for the recruitment of cognate binding partners. They also show a reduction in helix H6, particularly in the bacteriophage-borne members of the family associated with the application of a tilt across the subunit-subunit interface, and in the relative rotation of the GTPase and C-terminal domains against one another, yielding twisted-helical protofilaments and filaments [6,7]. All three archaeal CetZ structures resolved to date possess only a single turn of H6, substantially shorter than in FtsZ and tubulin. Some TubZ proteins lack H6 altogether, with a similar structural volume to CetZs in this region. Another commonality among all TubZ and CetZ proteins is the elongated single $\alpha$-helical $\mathrm{C}$-terminal tail that proceeds along the axis of the protofilament and contacts the adjacent subunit, suggesting that these might have common functions (e.g. stabilising the protofilament). 


\section{Figure legends}

Fig. 1 Molecular Phylogeny of the tubulin superfamily in archaea

(A) Radial phylogram of the tubulin superfamily, generated (by FastTree [50]) from the conserved blocks of a multiple sequence alignment of 271 tubulin superfamily members from across the major archaeal taxa, including representatives of tubulin and FtsZ from eukaryotes and bacteria. Bootstrap support is shown for selected branches. The previously-described families are represented by colour, as shown. (B) Identification of the indicated protein families in currently-known major archaeal taxa. A filled circle indicates that a family member has been identified in at least one species of this taxon. Taxa shown in grey indicate Candidate taxa or taxa for which only incomplete or scaffold-status genome sequence data are available.

Fig. 2 FtsZ1 and cell division in $H$. volcanii

(A) 2D images of $H$. volcanii cells (phase-contrast and GFP fluorescence overlay) that are expressing FtsZ1-GFP. An approximately central band of fluorescence is clear in all cells of various shapes, and appears brighter in cells undergoing division constriction. The band is seen as a ring in 3D (not shown). (B) Examples of the $H$. volcanii cell-division defect. Cells were induced to express the FtsZ1.D250A protein, a GTPase-domain mutant that strongly inhibits division, during mid-log phase growth. Cells continue to grow in a $2 \mathrm{D}$ manner, but fail to divide, forming giant plate cells in culture. (after ref [18]).

\section{Fig. 3 Crystal structures of CetZ proteins.}

(A) The crystal structure of the prototypical CetZ (Haloferax volcanii CetZ1; PDB code 4B46) is shown with the secondary structural elements indicated according to the tubulin / FtsZ nomenclature [31]. The inset panels indicate; the reduction of $\alpha$-helix H6, helix H11 from a viewpoint rotated $180^{\circ}$, helix H9 and loop H1-S2 respectively. Structural superimpositions of CetZ2 upon; (B) $\alpha$-tubulin, (C) FtsZ, 
(D) Bacillus thuringiensis TubZ and (E) bacteriophage $\Phi \mathrm{KZ}$ TubZ are shown in the side panels. All full structures are shown from the same viewpoint in cartoon A) or ribbon (B-E) representation, with GTPase domains coloured green, activation domains coloured purple, the principal core helix, H7, linking the two elements coloured yellow and the remaining amino- and carboxy-terminal extensions to the canonical tubulin / FtsZ fold shown in reds and blues respectively.

\section{Fig. 4 CetZ subunit-subunit interactions and comparisons}

(A) The structure of a $H$. volcanii CetZ2 protofilament is shown according to an identical colour scheme and representation as that in Fig. 2. Protofilaments of tubulin (B), FtsZ (C) and bacteriophage $\Phi K Z$ TubZ (D) are shown for the purposes of comparison. The inset panel shows the interaction site of the carboxy-terminus of a crystallographically related molecule in the CetZ2 crystal structure, compared to the binding mode of taxol within the same site on $\beta$-tubulin. (E) The contribution to the polymerisation interface by $\mathrm{H} 11$ of CetZ compared to that of similar regions in tubulin and Bacillus thuringiensis TubZ. (F) Comparison of the lateral interactions between $H$. volcanii CetZ2 protofilaments and those between adjacent monomers within the crystal structure of Methanosaeta thermophila CetZ. 


\section{References}

1. Allers T, Ngo HP, Mevarech M, Lloyd RG (2004) Development of additional selectable markers for the halophilic archaeon Haloferax volcanii based on the leuB and trpA genes. Appl Environ Microbiol 70 (2):943-953

2. Allers T, Mevarech M (2005) Archaeal genetics - the third way. Nat Rev Genet 6 (1):58-73. doi:nrg1504 [pii]

$10.1038 / \mathrm{nrg} 1504$ [doi]

3. Allers T, Barak S, Liddell S, Wardell K, Mevarech M (2010) Improved strains and plasmid vectors for conditional overexpression of His-tagged proteins in Haloferax volcanii. Appl Environ Microbiol 76 (6):1759-1769. doi:10.1128/aem.02670-09

4. Alushin GM, Lander GC, Kellogg EH, Zhang R, Baker D, Nogales E (2014) High-resolution microtubule structures reveal the structural transitions in alphabeta-tubulin upon GTP hydrolysis. Cell 157 (5):1117-1129. doi:10.1016/j.cell.2014.03.053

5. Andreu JM, Oliva MA, Monasterio O (2002) Reversible unfolding of FtsZ cell division proteins from archaea and bacteria. Comparison with eukaryotic tubulin folding and assembly. The Journal of biological chemistry 277 (45):43262-43270. doi:10.1074/jbc.M206723200

6. Aylett CH, Wang Q, Michie KA, Amos LA, Lowe J (2010) Filament structure of bacterial tubulin homologue TubZ. Proc Natl $\begin{array}{lllll}\text { Acad } & \text { Sci } & \text { USA } & 107 & \text { (46):19766-19771. }\end{array}$ doi:10.1073/pnas.1010176107

7. Aylett CH, Izore T, Amos LA, Lowe J (2013) Structure of the tubulin/FtsZ-like protein TubZ from Pseudomonas bacteriophage PhiKZ. J Mol Biol 425 (12):2164-2173. doi:10.1016/j.jmb.2013.03.019

8. Baker BJ, Saw JH, Lind AE, Lazar CS, Hinrichs K-U, Teske AP, Ettema TJG (2016) Genomic inference of the metabolism of cosmopolitan subsurface Archaea, Hadesarchaea. Nature Microbiology 1:16002. doi:10.1038/nmicrobiol.2016.2 http://www.nature.com/articles/nmicrobiol20162\#supplementaryinformation 
9. Baumann P, Jackson SP (1996) An archaebacterial homologue of the essential eubacterial cell division protein FtsZ. Proc Natl Acad Sci USA 93 (13):6726-6730

10. Bolhuis H, Palm P, Wende A, Falb M, Rampp M, Rodriguez-Valera F, Pfeiffer F, Oesterhelt D (2006) The genome of the square archaeon Haloquadratum walsbyi : life at the limits of water activity. BMC Genomics 7:169. doi:10.1186/1471-2164-7-169 11. Bult CJ, White O, Olsen GJ, Zhou L, Fleischmann RD, Sutton GG, Blake JA, FitzGerald LM, Clayton RA, Gocayne JD, Kerlavage AR, Dougherty BA, Tomb JF, Adams MD, Reich CI, Overbeek R, Kirkness EF, Weinstock KG, Merrick JM, Glodek A, Scott JL, Geoghagen NS, Venter JC (1996) Complete genome sequence of the methanogenic archaeon, Methanococcus jannaschii. Science 273 (5278):1058-1073

12. Burns DG, Janssen PH, Itoh T, Kamekura M, Li Z, Jensen G, Rodriguez-Valera F, Bolhuis H, Dyall-Smith ML (2007) Haloquadratum walsbyi gen. nov., sp. nov., the square haloarchaeon of Walsby, isolated from saltern crystallizers in Australia and Spain. Int J Syst Evol Microbiol 57 (Pt 2):387-392. doi:10.1099/ijs.0.64690-0

13. Buske PJ, Levin PA (2012) Extreme C terminus of bacterial cytoskeletal protein FtsZ plays fundamental role in assembly independent of modulatory proteins. The Journal of biological chemistry 287 (14):10945-10957. doi:10.1074/jbc.M111.330324

14. Buske PJ, Mittal A, Pappu RV, Levin PA (2015) An intrinsically disordered linker plays a critical role in bacterial cell division. Semin Cell Dev Biol 37:3-10. doi:10.1016/j.semcdb.2014.09.017

15. Chinen A, Uchiyama I, Kobayashi I (2000) Comparison between Pyrococcus horikoshii and Pyrococcus abyssi genome sequences reveals linkage of restriction-modification genes with large genome polymorphisms. Gene 259 (1-2):109-121

16. Dai K, Lutkenhaus J (1992) The proper ratio of FtsZ to FtsA is required for cell division to occur in Escherichia coli. J Bacteriol 174 (19):6145-6151

17. Diaz JF, Kralicek A, Mingorance J, Palacios JM, Vicente M, Andreu JM (2001) Activation of cell division protein FtsZ. Control of switch loop T3 conformation by the nucleotide gamma- 
phosphate. The Journal of biological chemistry 276 (20):1730717315. doi:10.1074/jbc.M010920200

18. Duggin IG, Aylett CH, Walsh JC, Michie KA, Wang Q, Turnbull L, Dawson EM, Harry EJ, Whitchurch CB, Amos LA, Lowe J (2015) CetZ tubulin-like proteins control archaeal cell shape. Nature 519 (7543):362-365. doi:10.1038/nature13983

19. Evans P (2006) Scaling and assessment of data quality. Acta $\begin{array}{llllll}\text { Crystallogr D Biol Crystallogr } 62 \quad(\mathrm{Pt} & 1): 72-82 .\end{array}$ doi:S0907444905036693 [pii]

$10.1107 / \mathrm{S} 0907444905036693$

20. Gilson PR, Beech PL (2001) Cell division protein FtsZ: running rings around bacteria, chloroplasts and mitochondria. Res Microbiol 152 (1):3-10

21. Huecas S, Andreu JM (2004) Polymerization of nucleotidefree, GDP- and GTP-bound cell division protein FtsZ: GDP makes the difference. FEBS Lett 569 (1-3):43-48. doi:10.1016/j.febslet.2004.05.048

22. Kawarabayasi Y, Sawada M, Horikawa H, Haikawa Y, Hino Y, Yamamoto S, Sekine M, Baba S, Kosugi H, Hosoyama A, Nagai Y, Sakai M, Ogura K, Otsuka R, Nakazawa H, Takamiya M, Ohfuku Y, Funahashi T, Tanaka T, Kudoh Y, Yamazaki J, Kushida N, Oguchi A, Aoki K, Kikuchi H (1998) Complete sequence and gene organization of the genome of a hyper-thermophilic archaebacterium, Pyrococcus horikoshii OT3. DNA Res 5 (2):55-76 23. Klenk HP, Clayton RA, Tomb JF, White O, Nelson KE, Ketchum KA, Dodson RJ, Gwinn M, Hickey EK, Peterson JD, Richardson DL, Kerlavage AR, Graham DE, Kyrpides NC, Fleischmann RD, Quackenbush J, Lee NH, Sutton GG, Gill S, Kirkness EF, Dougherty BA, McKenney K, Adams MD, Loftus B, Peterson S, Reich CI, McNeil LK, Badger JH, Glodek A, Zhou L, Overbeek R, Gocayne JD, Weidman JF, McDonald L, Utterback T, Cotton MD, Spriggs T, Artiach P, Kaine BP, Sykes SM, Sadow PW, D'Andrea KP, Bowman C, Fujii C, Garland SA, Mason TM, Olsen GJ, Fraser CM, Smith HO, Woese CR, Venter JC (1997) The complete genome sequence of the hyperthermophilic, sulphatereducing archaeon Archaeoglobus fulgidus. Nature 390 (6658):364370. doi: $10.1038 / 37052$ 
24. Kraemer JA, Erb ML, Waddling CA, Montabana EA, Zehr EA, Wang H, Nguyen K, Pham DS, Agard DA, Pogliano J (2012) A phage tubulin assembles dynamic filaments by an atypical mechanism to center viral DNA within the host cell. Cell 149 (7):1488-1499. doi:10.1016/j.cell.2012.04.034

25. Large A, Stamme C, Lange C, Duan Z, Allers T, Soppa J, Lund PA (2007) Characterization of a tightly controlled promoter of the halophilic archaeon Haloferax volcanii and its use in the analysis of the essential cct1 gene. Molecular microbiology 66 (5):10921106. doi:MMI5980 [pii]

10.1111/j.1365-2958.2007.05980.x [doi]

26. Larsen RA, Cusumano C, Fujioka A, Lim-Fong G, Patterson P, Pogliano J (2007) Treadmilling of a prokaryotic tubulin-like protein, TubZ, required for plasmid stability in Bacillus thuringiensis. Genes Dev 21 (11):1340-1352. doi:10.1101/gad.1546107

27. Lindas AC, Karlsson EA, Lindgren MT, Ettema TJ, Bernander R (2008) A unique cell division machinery in the Archaea. Proc Natl Acad Sci USA 105 (48):18942-18946

28. Lowe J, Amos LA (1998) Crystal structure of the bacterial cell-division protein FtsZ. Nature 391 (6663):203-206. doi: $10.1038 / 34472$

29. Lowe J, Amos LA (1999) Tubulin-like protofilaments in Ca2+-induced FtsZ sheets. Embo J 18 (9):2364-2371. doi:10.1093/emboj/18.9.2364

30. Lowe J, Amos LA (2000) Helical tubes of FtsZ from Methanococcus jannaschii. Biol Chem 381 (9-10):993-999. doi:10.1515/BC.2000.122

31. Lowe J, Li H, Downing KH, Nogales E (2001) Refined structure of alpha/beta-tubulin at $3.5 \AA$ resolution. J Mol Biol 313 (5):1045-1057. doi:10.1006/jmbi.2001.5077

32. Lowe J, Amos LA (2009) Evolution of cytomotive filaments: the cytoskeleton from prokaryotes to eukaryotes. International journal of biochemistry and cell biology 41 (2):323-329

33. Makarova KS, Koonin EV (2010) Two new families of the FtsZ-tubulin protein superfamily implicated in membrane remodeling in diverse bacteria and archaea. Biol Direct 5:33. doi:10.1186/1745-6150-5-33 
34. Margolin W, Wang R, Kumar M (1996) Isolation of an ftsZ homolog from the archaebacterium Halobacterium salinarium: implications for the evolution of FtsZ and tubulin. J Bacteriol 178 (5):1320-1327

35. Mendieta J, Rico AI, Lopez-Vinas E, Vicente M, Mingorance J, Gomez-Puertas P (2009) Structural and functional model for ionic $(\mathrm{K}(+) / \mathrm{Na}(+))$ and $\mathrm{pH}$ dependence of GTPase activity and polymerization of FtsZ, the prokaryotic ortholog of tubulin. J Mol Biol 390 (1):17-25. doi:10.1016/j.jmb.2009.05.018

36. Monahan LG, Robinson A, Harry EJ (2009) Lateral FtsZ association and the assembly of the cytokinetic $\mathrm{Z}$ ring in bacteria. Molecular microbiology 74 (4):1004-1017. doi:MMI6914 [pii] $10.1111 / \mathrm{j} .1365-2958.2009 .06914 . x$ [doi]

37. Mukherjee A, Lutkenhaus J (1998) Dynamic assembly of FtsZ regulated by GTP hydrolysis. Embo J 17 (2):462-469. doi:10.1093/emboj/17.2.462

38. Mullakhanbhai MF, Larsen H (1975) Halobacterium volcanii spec. nov., a Dead Sea halobacterium with a moderate salt requirement. Arch Microbiol 104 (3):207-214

39. Nagahisa K, Nakamura T, Fujiwara S, Imanaka T, Takagi M (2000) Characterization of FtsZ homolog from hyperthermophilic archaeon Pyrococcus kodakaraensis KOD1. J Biosci Bioeng 89 (2):181-187

40. Ng KH, Srinivas V, Srinivasan R, Balasubramanian M (2013) The Nitrosopumilus maritimus CdvB, but not FtsZ, assembles into polymers. 2013:104147. doi:10.1155/2013/104147

41. Ng WV, Kennedy SP, Mahairas GG, Berquist B, Pan M, Shukla HD, Lasky SR, Baliga NS, Thorsson V, Sbrogna J, Swartzell S, Weir D, Hall J, Dahl TA, Welti R, Goo YA, Leithauser B, Keller K, Cruz R, Danson MJ, Hough DW, Maddocks DG, Jablonski PE, Krebs MP, Angevine CM, Dale H, Isenbarger TA, Peck RF, Pohlschroder M, Spudich JL, Jung KW, Alam M, Freitas T, Hou S, Daniels CJ, Dennis PP, Omer AD, Ebhardt H, Lowe TM, Liang P, Riley M, Hood L, DasSarma S (2000) Genome sequence of Halobacterium species NRC-1. Proc Natl Acad Sci USA 97 (22):12176-12181. doi:10.1073/pnas. 190337797 
42. Nogales E, Downing KH, Amos LA, Lowe J (1998) Tubulin and FtsZ form a distinct family of GTPases. Nature Structural Biology 5 (6):451-458

43. Nogales E, Wolf SG, Downing KH (1998) Structure of the alpha/beta-tubulin dimer by electron crystallography. Nature 391 (6663):199-203. doi:10.1038/34465

44. Nunoura T, Takaki Y, Kakuta J, Nishi S, Sugahara J, Kazama H, Chee GJ, Hattori M, Kanai A, Atomi H, Takai K, Takami H (2011) Insights into the evolution of Archaea and eukaryotic protein modifier systems revealed by the genome of a novel archaeal group. Nucleic Acids Res 39 (8):3204-3223. doi:10.1093/nar/gkq1228

45. Oliva MA, Cordell SC, Lowe J (2004) Structural insights into FtsZ protofilament formation. Nature Structural and Molecular Biology 11 (12):1243-1250. doi:10.1038/nsmb855

46. Oliva MA, Trambaiolo D, Lowe J (2007) Structural insights into the conformational variability of FtsZ. J Mol Biol 373 (5):12291242. doi:10.1016/j.jmb.2007.08.056

47. Ozawa K, Harashina T, Yatsunami R, Nakamura S (2005) Gene cloning, expression and partial characterization of cell division protein FtsZ1 from extremely halophilic archaeon Haloarcula japonica strain TR-1. Extremophiles 9 (4):281-288. doi:10.1007/s00792-005-0443-6

48. Pelve EA, Lindas AC, Martens-Habbena W, de la Torre JR, Stahl DA, Bernander R (2011) Cdv-based cell division and cell cycle organization in the thaumarchaeon Nitrosopumilus maritimus. Molecular microbiology 82 (3):555-566. doi:10.1111/j.13652958.2011.07834.x

49. Poplawski A, Gullbrand B, Bernander R (2000) The ftsZ gene of Haloferax mediterranei: sequence, conserved gene order, and visualization of the FtsZ ring. Gene 242 (1-2):357-367. doi:S0378-1119(99)00517-X [pii]

50. Price MN, Dehal PS, Arkin AP (2010) FastTree 2-approximately maximum-likelihood trees for large alignments. PLoS One 5 (3):e9490. doi:10.1371/journal.pone.0009490

51. Reed CJ, Lewis H, Trejo E, Winston V, Evilia C (2013) Protein adaptations in archaeal extremophiles. 2013:373275. doi: $10.1155 / 2013 / 373275$ 
52. Reuter CJ, Maupin-Furlow JA (2004) Analysis of proteasome-dependent proteolysis in Haloferax volcanii cells, using short-lived green fluorescent proteins. Appl Environ Microbiol 70 (12):7530-7538. doi:10.1128/AEM.70.12.7530-7538.2004

53. Richards KL, Anders KR, Nogales E, Schwartz K, Downing $\mathrm{KH}$, Botstein D (2000) Structure-function relationships in yeast tubulins. Molecular biology of the cell 11 (5):1887-1903

54. Samson RY, Obita T, Freund SM, Williams RL, Bell SD (2008) A role for the ESCRT system in cell division in archaea. Science 322 (5908):1710-1713

55. Samson RY, Bell SD (2009) Ancient ESCRTs and the evolution of binary fission. Trends Microbiol 17 (11):507-513. doi:10.1016/j.tim.2009.08.003

56. Scheffers DJ, de Wit JG, den Blaauwen T, Driessen AJ (2002) GTP hydrolysis of cell division protein FtsZ: evidence that the active site is formed by the association of monomers. Biochemistry 41 (2):521-529. doi:bi011370i [pii]

57. Seitz KW, Lazar CS, Hinrichs KU, Teske AP, Baker BJ (2016) Genomic reconstruction of a novel, deeply branched sediment archaeal phylum with pathways for acetogenesis and sulfur reduction. Isme J. doi:10.1038/ismej.2015.233

58. Spang A, Saw JH, Jorgensen SL, Zaremba-Niedzwiedzka K, Martijn J, Lind AE, van Eijk R, Schleper C, Guy L, Ettema TJ (2015) Complex archaea that bridge the gap between prokaryotes and eukaryotes. Nature 521 (7551):173-179. doi:10.1038/nature14447

59. Stoeckenius W (1981) Walsby's square bacterium: fine structure of an orthogonal procaryote. J Bacteriol 148 (1):352-360

60. Sun Q, Margolin W (1998) FtsZ dynamics during the division cycle of live Escherichia coli cells. J Bacteriol 180 (8):2050-2056

61. Tan CM, Therien AG, Lu J, Lee SH, Caron A, Gill CJ, Lebeau-Jacob C, Benton-Perdomo L, Monteiro JM, Pereira PM, Elsen NL, Wu J, Deschamps K, Petcu M, Wong S, Daigneault E, Kramer S, Liang L, Maxwell E, Claveau D, Vaillancourt J, Skorey K, Tam J, Wang H, Meredith TC, Sillaots S, Wang-Jarantow L, Ramtohul Y, Langlois E, Landry F, Reid JC, Parthasarathy G, Sharma S, Baryshnikova A, Lumb KJ, Pinho MG, Soisson SM, 
Roemer T (2012) Restoring methicillin-resistant Staphylococcus aureus susceptibility to beta-lactam antibiotics. Sci Transl Med 4 (126):126ra135. doi:10.1126/scitranslmed.3003592

62. Vaughan S, Wickstead B, Gull K, Addinall SG (2004) Molecular evolution of FtsZ protein sequences encoded within the genomes of archaea, bacteria, and eukaryota. J Mol Evol 58 (1):1929. doi:10.1007/s00239-003-2523-5 [doi]

63. Walsby AE (1980) Square bacterium. Nature 283 (5742):6971. doi:10.1038/283069a0

64. Wang X, Lutkenhaus J (1996) FtsZ ring: the eubacterial division apparatus conserved in archaebacteria. Molecular microbiology 21 (2):313-319

65. Webster G, O'Sullivan LA, Meng Y, Williams AS, Sass AM, Watkins AJ, Parkes RJ, Weightman AJ (2015) Archaeal community diversity and abundance changes along a natural salinity gradient in estuarine sediments. FEMS Microbiol Ecol 91 (2):1-18. doi:10.1093/femsec/fiu025

66. Yaoi T, Laksanalamai P, Jiemjit A, Kagawa HK, Alton T, Trent JD (2000) Cloning and characterization of ftsZ and pyrF from the archaeon Thermoplasma acidophilum. Biochem Biophys Res Commun 275 (3):936-945. doi:10.1006/bbrc.2000.3401 [doi]

S0006-291X(00)93401-6 [pii]

67. Yutin N, Koonin EV (2012) Archaeal origin of tubulin. Biol Direct 7:10. doi:10.1186/1745-6150-7-10

1745-6150-7-10 [pii]

68. Zhalnina KV, Dias R, Leonard MT, Dorr de Quadros P, Camargo FA, Drew JC, Farmerie WG, Daroub SH, Triplett EW (2014) Genome sequence of Candidatus Nitrososphaera evergladensis from group I.1b enriched from Everglades soil reveals novel genomic features of the ammonia-oxidizing archaea. PLoS One 9 (7):e101648. doi:10.1371/journal.pone.0101648 


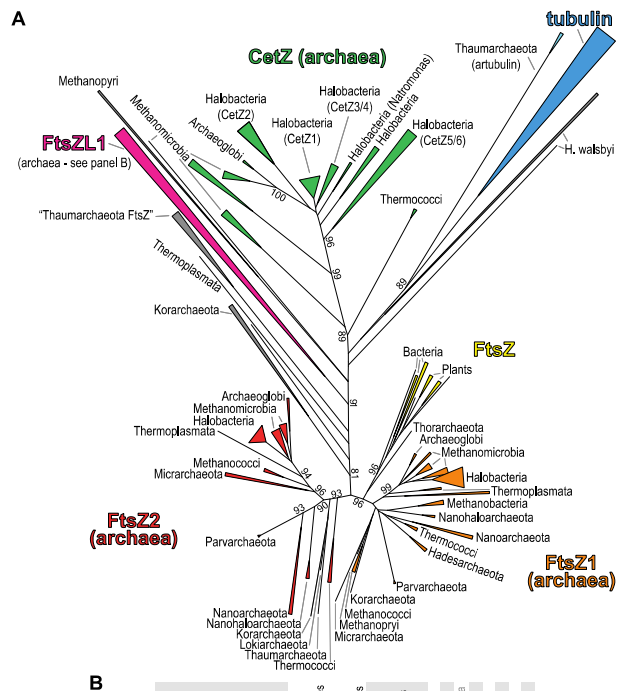

B

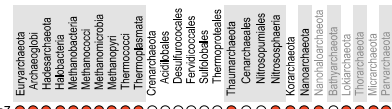

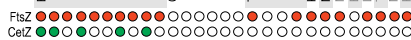

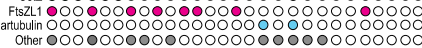




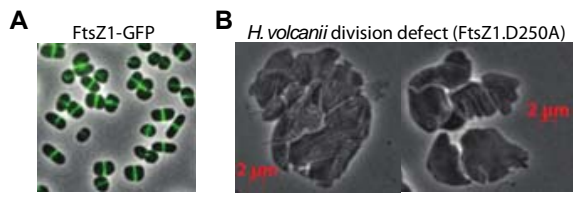



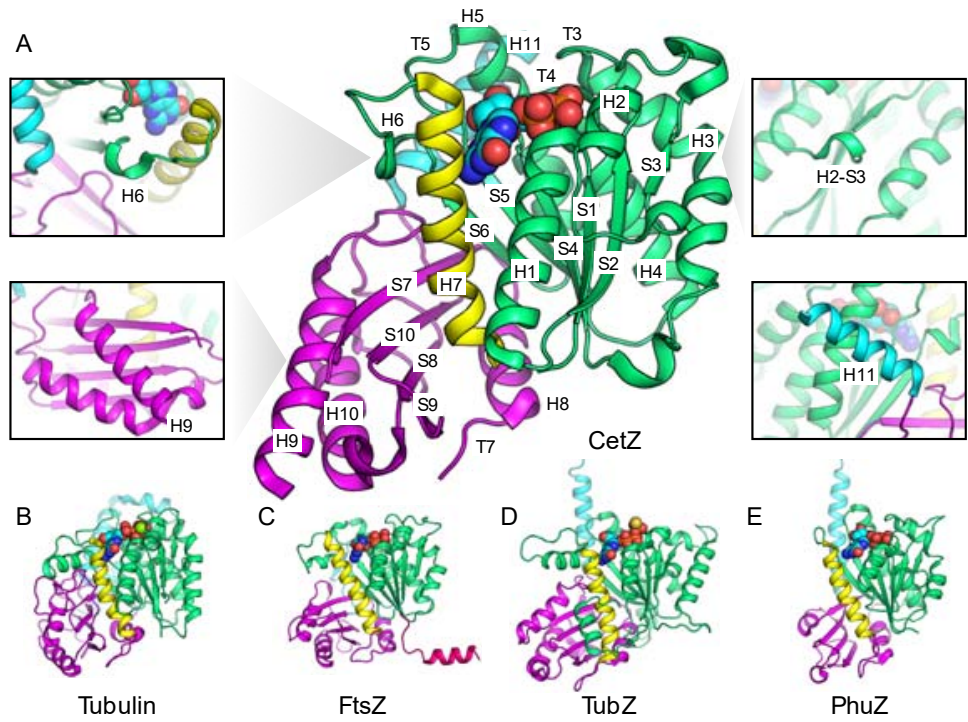

Tubulin

FtsZ 


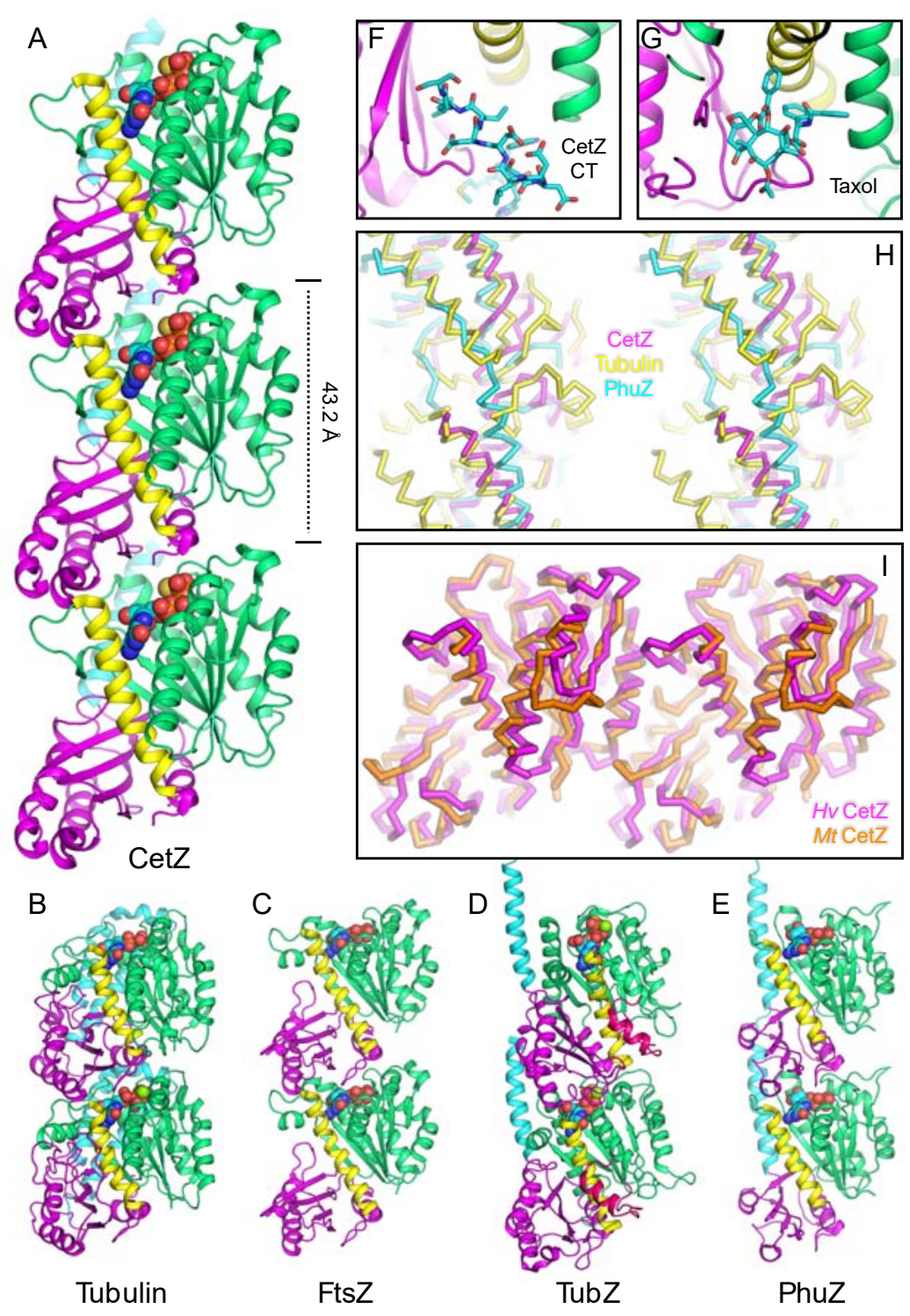

